\title{
EXPERIÊNCIAS DE ACOLHIMENTO SEGUNDO PROFISSIONAIS DE UM CENTRO DE ATENÇÃO PSICOSSOCIAL
}

\section{EXPERIENCES OF EMBRACEMENT ACCORDING TO PROFESSIONALS OF A PSYCHOSOCIAL ATTENTION CENTER}

\section{ACOGIMIENTO SEGUNDO PROFESIONALES DE UN CENTRO DE ATENCIÓN PSICOSOCIAL}

\author{
Renata Fabiana Pegoraro ${ }^{1}$, Ludmilla Suellen Nunes Bastos ${ }^{2}$
}

\section{RESUMO}

Objetivos: Este artigo teve como objetivo identificar as concepções sobre o processo de acolhimento realizado em um Centro de Atenção Psicossocial segundo seus profissionais. Método: A pesquisa realizada foi qualitativa, de natureza descritiva, e fez uso de um roteiro de entrevistas, aplicado em quatro profissionais, sobre o funcionamento do CAPS e os cuidados prestados, além de observação participante. As entrevistas foram transcritas e submetidas à análise de conteúdo. Resultados: Os participantes destacaram mudanças na forma de realizar o acolhimento a partir de mudanças na demanda do CAPS, bem como apresentaram pontos positivos e dificuldades da realização de um grupo de acolhimento. Conclusão: Destaca-se a aproximação de familiares-usuários-equipe a partir do grupo de acolhimento, pelo desenvolvimento de escuta e vínculo, bem como o funcionamento deste grupo como forma de minimizar dificuldades advindas de falhas na rede de saúde mental local.

Palavras chaves: Acolhimento, Saúde Mental, Serviços de Saúde.

\section{ABSTRACT}

Objectives: This article aims to identify the conceptions of the embracement procedure performed in a Psychosocial Care Center according to its professionals. Method: The research was qualitative, descriptive, and made use of a set of interviews, applied to four professionals on the functioning of the CAPS and the care provided in addition to participant observation. The interviews were transcribed and subjected to content analysis. Results: The participants highlighted changes in the way of carrying out the host from changes in demand CAPS and showed strengths and difficulties of performing a host group. Conclusion: The study highlights the approach of family-members-team from the host group, the development of listening and bonding, as well as the operation of this group in order to minimize difficulties arising from flaws in the local mental health system.

Keywords: User Embracement, Mental Health, Health Services.

\footnotetext{
${ }^{1}$ Doutora em Psicologia pela USP de Ribeirão Preto - SP, Especialista em Saúde Coletiva - UFSCAR, Instituto de Psicologia - UFU, Brasil. E-mail: rfpegoraro@yahoo.com.br

${ }^{2}$ Psicóloga pela Pontifícia Universidade Católica de Goiás. E-mail: ludnunes.psi@ gmail.com
} 


\section{RESUMEN}

Objetivos: Este artículo tiene como objetivo identificar las concepciones del procedimiento de recepción realizado en un Centro de Atención Psicosocial de acuerdo a sus profesionales. Método: La investigación fue cualitativa, descriptiva, e hizo uso de una serie de entrevistas, aplicado a cuatro profesionales sobre el funcionamiento de los CAPS y la atención recibida, además de la observación participante. Las entrevistas fueron transcritas y sometidas a análisis de contenido. Resultados: Los participantes destacaron los cambios en la forma de llevar a cabo la acogida de los cambios en la demanda y CAPS mostraron fortalezas y dificultades de la realización de un grupo de hosts. Conclusión: El estudio pone de relieve el enfoque de la familia-miembros-equipo del grupo de acogida, el desarrollo de la escucha y la unión, así como la operación de este grupo con el fin de reducir al mínimo las dificultades derivadas de fallas en el sistema de salud mental local.

Palavras clave: Acogimiento, Salud Mental, Servicios de Salud.

\section{INTRODUÇÃO}

O Centro de Atenção Psicossocial (CAPS) é um serviço de saúde mental que oferece atendimento a pessoas em situação de sofrimento psíquico grave e/ou decorrente do uso de substâncias e visa a inserção social e cultural de usuários e seus familiares no território. Atualmente, o CAPS integra a Rede de Atenção Psicossocial e além de oferecer atendimentos diários aos usuários, é também responsável, por gerenciar projetos terapêuticos personalizados para cada usuário, organizar a rede de serviços de saúde mental no território e dar suporte aos profissionais de saúde mental da rede básica. ${ }^{1}$

Além do acompanhamento clínico, os CAPS oferecem outras atividades que promovem a reinserção social e reabilitação psicossocial, tais como oficinas terapêuticas, atividades comunitárias e artísticas, psicoterapia individual ou em grupo, orientação e acompanhamento do uso de medicação, visitas e atendimentos domiciliares. ${ }^{1}$

Além de todas essas atividades, o acolhimento também é uma importante ferramenta adotada pelas equipes dos CAPS, sendo realizada diariamente pela equipe. No processo de acolhimento, a equipe prioriza a escuta qualificada que propicia a formação de vínculo do usuário com o serviço de saúde à medida que se passa a enxergar a pessoa e não a sua doença. ${ }^{2}$ Não se define acolhimento como um espaço ou um local, com horário ou profissional específico para realizá-lo, mas sim uma atitude acolhedora por parte de todos os profissionais, que "escutam e abrigam" aquele que busca auxílio para suas demandas. E por esse motivo pode ser 
compreendido como uma ação que deve ocorrer em todos os locais e momentos do serviço. ${ }^{3}$ Além disso, o acolhimento é uma tecnologia para reorganização dos serviços, que visa garantir acesso universal e humanizado aos equipamentos de saúde, a reorganização dos processos de trabalho e o acesso com responsabilização e resolutividade. ${ }^{4-5}$

Ainda que a literatura ${ }^{4}$ aponte para uma atitude acolhedora diária dirigida pela equipe aos usuários, um estudo brasileiro realizado a partir de entrevistas com profissionais permitiu o delineamento de diferentes compreensões sobre a ferramenta "Acolhimento", a saber: (a) Acolhimento como uma entrevistatriagem, como a porta de entrada do serviço; ( b ) Acolhimento enquanto ação em saúde, sinônimo de "ato de acolher", que pode ocorrer em diferentes espaços e momentos da rotina dos CAPS e permitem relações mais humanizadas entre usuários e trabalhadores; ( c ) Acolhimento como dispositivo-analisador do processo de trabalho em saúde, isto é, como um conceito empregado para problematizar a assistência prestada aos usuários; ( d ) Acolhimento como um espaço de escuta, processo que permite visualizar o estabelecimento de confiança dos usuários nos técnicos, aproximando-se do conceito winnicotiano de holding, ou seja, um ambiente suficientemente-bom, capaz de sustentar o desenvolvimento pessoal por meio das ações da equipe.

A partir do reconhecimento da importância do acolhimento nos âmbito da rede SUS $^{6}$ e de seu emprego nos CAPS ${ }^{1}$, sendo apontado como diretriz pelo Ministério da Saúde, bem como da diversidade de pontos de vista sobre esta estratégia entre profissionais da rede, colocaram-se como problemas de pesquisa: de que modo é realizado o acolhimento em CAPS atualmente? Há uma única forma adotada pela equipe? Há necessidade de mudanças na forma como o acolhimento tem sido realizado? Quais aspectos positivos $\mathrm{e}$ as dificuldades que esta ferramenta gera?

A partir destas questões, foi objetivo da presente pesquisa identificar as concepções de profissionais de um Centro de Atenção Psicossocial sobre o acolhimento.

\section{MÉTODO}

O estudo foi desenvolvido como pesquisa qualitativa de natureza descritiva. Foi utilizado roteiro de entrevista semiestruturado composto inicialmente pelos 
seguintes dados sócio demográficos: idade, sexo, estado civil, formação profissional (graduação, especialização) e tempo de atuação no serviço de saúde. Foram investigadas, ainda, as seguintes questões norteadoras: experiências de atuação anterior em serviços de saúde/saúde mental; atividades desenvolvidas na atualidade no CAPS; concepções sobre o acolhimento; avaliação sobre os usuários do CAPS (principais diagnósticos, avanços e dificuldades encontradas para executar o trabalho no dia-a-dia). Além das entrevistas, foi realizada observação participante das atividades referentes ao acolhimento dos usuários. Foram realizadas quatro visitas ao CAPS com esta finalidade, cada uma com 3 horas de duração.

Participaram do estudo quatro profissionais de um CAPS II da cidade de Goiânia, que atendia usuários com transtornos mentais severos e persistentes. Foram utilizados os seguintes critérios de inclusão: trabalhar no CAPS há mais de seis meses, ser maior de 18 anos, ter disponibilidade para ceder entrevista gravada e realizar o acolhimento aos usuários no período em que a coleta ocorreu.

Todos os participantes do estudo receberam um Termo de Consentimento
Livre e Esclarecido (TCLE), que lhes assegurava o sigilo das informações declaradas e a preservação da identidade dos entrevistados, conforme aprovado pelo Comitê de Ética em Pesquisa com Seres Humanos da PUC Goiás (Parecer CEP/SGC 1840/2011). Para assegurar o anonimato, foram utilizadas as denominações E1, E2, E3 e E4 para identificação dos entrevistados.

As entrevistas ocorreram no próprio CAPS, no ano de 2012, em uma sala reservada e no horário escolhido pelo participante. Cada entrevista foi transcrita na íntegra e o conjunto das transcrições foi submetida à análise de conteúdo. ${ }^{7}$

\section{RESULTADOS}

Todos os entrevistados eram do sexo feminino, com idade entre 20 e 50 anos e concursadas junto à prefeitura municipal; duas tinham companheiro e as outras eram solteiras. As entrevistadas trabalhavam no CAPS de 5 a 10 anos e todas haviam realizado uma especialização (saúde mental ou outras áreas).

Categoria 1 - Diferentes formatos do Acolhimento na história do CAPS 
De acordo com os profissionais, o acolhimento era feito inicialmente durante toda a semana pelos profissionais do CAPS junto ao usuário que pela primeira vez chegava ao serviço, com a coleta de algumas informações sobre sua história e os motivos pelos quais foi ao CAPS. O acolhimento ocorria sem a necessidade de agendamento e era realizado por um profissional apenas por turno (um profissional pela manhã e outro à tarde, conforme escala) e todos os casos eram discutidos na reunião geral de equipe (semanal) para elaboração do projeto terapêutico do usuário:

O acolhimento era feito durante a semana... na sexta feira eram levados para reunião de equipe...para as pessoas decidirem qual seria o projeto terapêutico para a pessoa (E4).

A demanda do CAPS foi crescendo e o número de profissionais que integrava a equipe também. Segundo os relatos, o acolhimento transformou-se em atividade que causava sobrecarga, pela gravidade de alguns casos e pela necessidade de um "segundo olhar" dirigido ao usuário e sua história, antes de se definir se o usuário deveria ou não ser inserido na rotina do CAPS. Partindo dessas dificuldades, o acolhimento começou a ser feito em dupla de profissionais:
Com o tempo...o CAPS foi tendo uma demanda maior...e aí quase no mesmo instante teve que haver uma maneira de se fazer acolhimento em dupla também. (E4)

Segundo os entrevistados, em um terceiro momento, houve nova alteração na forma como o acolhimento era realizado. Além do acolhimento individual ao usuário que procurava o CAPS, foi criado pela equipe o "Grupo de Acolhimento" com o intuito de conhecer melhor os usuários, suas demandas, e procurar esclarecer os questionamentos que estes traziam.

No momento em que ocorreu a coleta de dados, ao procurar o CAPS, segundo os entrevistados, a pessoa poderia fazê-lo espontaneamente ou por meio de algum encaminhamento. Ela seria acolhida por um dos profissionais, que faria uma escuta, orientaria quanto ao atendimento e funcionamento do CAPS, e preencheria uma ficha com a história de vida e clínica do usuário. A partir desse momento, o usuário era convidado a participar do "Grupo de Acolhimento", cujas atividades ocorriam duas vezes por semana. Esse grupo de acolhimento permitiria atender a necessidade de maiores esclarecimentos sobre o funcionamento do CAPS aos usuários e proporcionar um ambiente para conhecê-los melhor. 
Às vezes [o usuário] começava a frequentar [as atividades do CAPS] e via que não era aquilo, ou às vezes aquilo que você estava propondo não tinha a ver com aquela pessoas ou às vezes nem vinha... vinha somente para o acolhimento...aí se pensou...vamos fazer um grupo para que a pessoa tenha um tempo para conhecer o serviço e a equipe fica conhecendo a pessoa para fazer um projeto mais pertinente...e aí foi por isso que foi criado esse grupo de acolhimento. (E2)

Categoria 2 - Funções do Acolhimento e do Grupo de Acolhimento

Estabeleceram-se no CAPS dois momentos distintos, o Acolhimento e o Grupo de Acolhimento. Um dos entrevistados destacou que:

É no acolhimento que a gente pega vários dados, desde a gestação da pessoa, o que levou a aquela doença, quantas internações ela já passou, tudinho... então é o primeiro momento quando a pessoa vem a unidade [...] Aí a partir desse acolhimento o profissional que faz o [grupo de acolhimento] ele também já faz uma pequena avaliação se essa pessoa vai se beneficiar no CAPS ou no ambulatório. (E2)

Após o acolhimento, o usuário era convidado para participar do grupo. Os profissionais apontaram que o Grupo de Acolhimento tinha como função avaliar o usuário, permitir a vinculação usuárioequipe, filtrar usuários com ou sem perfil para o CAPS, promover a escuta e aumentar a adesão ao tratamento no CAPS. No Grupo de Acolhimento os profissionais teriam maior oportunidade para conhecer o usuário, sua história de vida, para assim avaliar se este tinha perfil ou não para o serviço. Assim, a função de "filtrar" era muito importante, como apontou um entrevistado:

[...] no grupo de acolhimento que a gente vai avaliar se realmente é caso para ambulatório [de psiquiatria] ou para cá... (E1)

A maior parte dos usuários que frequentava o grupo permanecia de 2 a 3 meses, ainda que não houvesse um tempo mínimo ou máximo, sendo sempre avaliada a demanda de cada um. Nesse período, o usuário frequentava apenas esse grupo (reunião com outros usuários sob coordenação de um profissional e avaliação médica). Se o usuário tivesse perfil para ser atendido no CAPS, os profissionais responsáveis pelo grupo encaminhariam o mesmo a uma das mini equipe de referência do serviço para dar continuidade ao atendimento e condução do plano terapêutico personalizado. Caso não tivesse perfil, era feito o encaminhamento para outro serviço da rede:

Mas a princípio você vai...a gente vai conhece você, você vai trazer sua história no 
grupo, quem é você, os coordenadores vão estar observando quem é aquele que está ali no grupo. E aí, a partir de cada situação, cada história, a gente pode direcionar na semana seguinte para uma mini equipe ou na semana seguinte pra um serviço da rede de contato... (E1)

Dentre os usuários que frequentavam o Grupo de Acolhimento no momento das entrevistas, encontravam-se aqueles que chegavam em crise, com depressão de moderada a leve, quadros de esquizofrenia, transtorno bipolar, de ansiedade, certos tipos de fobia, pacientes crônicos, ou até mesmo com um transtorno orgânico leve ou grave:

Mas a gente precisa entender que esse grupo de acolhimento ele abrange usuários que estão em crise, alguma depressão mais moderada, leve, mais grave, pessoas que simplesmente tem...é crônico, que as vezes não consegue nem ta aqui, que, a gente tem esse perfil...então tem pessoas com um transtorno mais leve, uma fobia, um transtorno de ansiedade. (E4)

Um entrevistado ilustrou o funcionamento do Grupo de Acolhimento, cuja função final seria encaminhar $o$ usuário para seguimento de uma das miniequipes de referência ou para outro serviço da rede.

Categoria 3 - Aspectos positivos e dificuldades do grupo de acolhimento
Apesar da denominação "Grupo de Acolhimento", adotada pelos entrevistados e demais profissionais do CAPS, funcionavam dois grupos: um dirigido a usuários e outro a familiares. Às terças e quintas no período da tarde, durante cerca de 2 horas, um profissional coordenava o "grupo de acolhimento para usuários" e outro profissional simultaneamente coordenava o "grupo de acolhimento para familiares". Ao todo, eram quatro os profissionais responsáveis por estes grupos e revezavam a coordenação dos mesmos durante o mês. Além disso, era agendada uma avaliação com um dos psiquiatras da unidade para o usuário.

Ao chegar no CAPS, as pessoas vinham acompanhadas de vizinhos, amigos, conhecidos, ou pessoas da famílias, e estas ficavam esperando enquanto os usuários eram atendidos. Desta forma, a equipe do CAPS criou um espaço para as famílias, com o intuito de vincular os familiares ao serviço e ajudar no tratamento. As reuniões com usuários e familiares do Grupo de Acolhimento aconteciam nos mesmos dias e horários:

E aí a gente começou a perceber que tinham pessoas que eram trazidas por familiares, mas ficavam aguardando...então vamos já aproveitar isso para já vincular os familiares ao serviço...onde começamos o grupo de família também. (E4) 
A inclusão semanal dos familiares nos atendimentos do CAPS foi avaliada positivamente pelos entrevistados. $\mathrm{O}$ Grupo de Acolhimento foi apontado como um dos espaços mais importantes dentro do serviço, porque era através dele que se formava o vínculo entre o CAPS e os usuários e as famílias. A partir do momento em que o usuário conhecia a fundo o funcionamento do serviço, ele teria maior adesão, e assim mais qualidade em seu tratamento:

Nesse grupo de acolhimento, a gente tenta falar de tudo o que circunda a vida desse usuário para a gente conhecer mesmo, qual o cotidiano dele... pra gente buscar atividades que ele vai ter essa adesão. (E3)

Deste modo, compreende-se que o Grupo de Acolhimento nutria os profissionais com informações para a elaboração do Projeto Terapêutico Individual dos usuários, em um segundo momento, quando deixavam de fazer parte deste grupo e eram encaminhados a uma mini-equipe de referência, integrando a "rotina" do CAPS.

Apesar dos pontos positivos destacados, segundo os entrevistados, parte dos usuários do grupo de acolhimento não tinha perfil para estar o CAPS e ali permaneciam por dificuldade de articulação de cuidado em outro ponto da rede. Deste modo, o grupo de acolhimento também supria uma necessidade de cuidar dos usuários em sofrimento, mas cujo perfil não era para o CAPS. De acordo com os profissionais, o encaminhamento de um usuário para o ambulatório municipal demorava cerca de três meses a um ano para sair, fazendo com que o CAPS ficasse superlotado:

Teve uma época que era muito crítico o encaminhamento para o ambulatório, por exemplo... para encaminhar para o ambulatório. Aí o serviço tava inchando, inchando, inchando... que a gente não conseguia encaminhar... (E4)

Pelo fato da demora no encaminhamento e não existir outras opções de serviços na rede que pudessem atender esses usuários, o CAPS acabava por suportar uma demanda excessiva, sem perfil para o cuidado neste equipamento:

Mas nós temos alguns problemas que estão ligados a problemas na nossa rede...então as vezes as pessoas vem em busca de um serviço como este por que não tem outro serviço na rede básica...então a pessoa vem para ser acolhida no CAPS.... pessoa não tem perfil de CAPS...ela não tem necessidade desse serviço...mas eu não tenho um serviço básico na rede que a atenda. (E4)

Esta situação, segundo os entrevistados, tornava o trabalho cansativo, desgastante e dificultava o 
acompanhamento dos usuários dentro do serviço:

É um pouco desgastante, em função assim que são " $n$ " situações que acontecem numa lista dessa...então vai ter aqueles que a gente conhece bem, que está tudo ok, direitinho dentro de um mês, dois, você já faz o projeto [terapêutico individualizado], tem aqueles que você sabe que tá, que é caso para ambulatório, tem aqueles que vem uma vez só e nunca mais voltou. (E3)

Se por um lado, havia a preocupação de manter o usuário no CAPS até que houvesse vaga no ambulatório municipal, por outro havia o reconhecimento de que muitos não frequentavam o grupo de acolhimento. A lista de usuários do grupo de acolhimento era de aproximadamente 150 usuários, mas frequentam o CAPS somente de 12 a 15 . Muitos não participavam efetivamente do grupo e isto gerava certa dificuldade no funcionamento do serviço. Um entrevistado exemplifica:

Não é caso de CAPS, mas precisa de um acompanhamento psiquiátrico. Mas não do tipo CAPS. Aí essa vaga que demora, eu mandar ela pra lá, demora de três meses... aí ela vai ficando [no grupo de acolhimento]... (E2)

Além das questões acima, os entrevistados colocaram também as dificuldades dos profissionais do grupo de acolhimento de se reunirem, discutirem os casos, realizar os encaminhamentos devidos e de trabalhar em coletivo. Diferente das outras mini-equipes que funcionavam no CAPS, os técnicos do grupo de acolhimento não tinham um dia da semana específico para se reunirem e discutirem os casos, sendo feito os encaminhamentos e projetos terapêuticos, individualmente e nem sempre com o consenso com os demais da equipe:

Mas você não tem todas as pessoas sentadas para discutir...muito raro, sabe! Tem pessoas que estão no grupo de acolhimento desde que ele foi criado e não consegue achar horário para sentar... essas pessoas todas... A barreira talvez seja a organização do tempo das pessoas... às vezes as pessoas tem dificuldade de trabalhar no coletivo... (E4).

Ao final da pesquisa, na devolutiva dos dados, no ano seguinte à coleta, a equipe informou que o grupo de acolhimento fora extinto, tendo sido avaliado pouco produtivo. $\mathrm{O}$ acolhimento individual permanecia e a equipe estava investindo na capacitação da atenção básica como forma de ampliar os pontos de cuidados de usuários em situação de sofrimento psíquico, mas cujo perfil não apontava a necessidade de permanência no CAPS.

\section{DISCUSSÃO}


No CAPS em que o estudo foi realizado, o acolhimento era feito, num primeiro momento, por um conjunto de profissionais que atendiam os usuários que procuravam o serviço. Era feita uma escuta, onde o profissional procurava entender a demanda do usuário e preencher uma ficha com a história de vida e história clínica deste, logo após, era avaliado pelo profissional se está pessoa iria se beneficiar das atividades do CAPS, ou não. Neste momento, o acolhimento era a porta de entrada do serviço ${ }^{4}$, isto é, tinha a função de triagem e também de espaço de escuta do usuário, para posterior discussão e elaboração de projeto terapêutico com a participação da equipe. Ao cumprir a função de nortear a construção do projeto terapêutico singular, o acolhimento é assumido como uma ferramenta que permite o diálogo entre profissionais e família para o cuidado do usuário que, nesse primeiro momento de ingresso no CAPS, encontra-se em crise ou situação de intenso sofrimento psíquico. ${ }^{6,14}$

Em um segundo momento, formouse o Grupo de Acolhimento, com parte dos profissionais (uma mini-equipe) do CAPS responsáveis pela realização de atividades grupais com usuários (Grupo de Usuários) e familiares (Grupo de Familiares). Ou seja, após o Acolhimento, o usuário era convidado para participar do Grupo de Acolhimento. Não havia um período mínimo ou máximo para permanência dos usuários neste Grupo e sua principal finalidade era avaliar se o usuário seria ou não caso para ser atendido no CAPS.

Neste momento, o Grupo de Acolhimento assumiu o caráter tanto de dispositivo-analisador do processo de trabalho em saúde quanto de espaço de escuta. $\mathrm{O}$ acolhimento enquanto espaço de escuta $^{4}$ pode proporcionar um ambiente suficientemente bom (holding) ao usuário e auxiliar no processo terapêutico. $\mathrm{O}$ acolhimento deve ser realmente eficaz, e para tal é preciso qualificar a escuta e construir juntamente com a equipe um modelo de assistência que seja centrada no usuário $^{8}$, e que procure dar respostas positivas aos problemas que estes trazem, mesmo que a resposta seja somente o ato de acolher.

O caráter de Analisador do processo de trabalho em saúde ${ }^{4}$ assenta-se no acolhimento como uma perspectiva problematizadora da assistência prestada aos usuários, ou seja, ele gera dados, por exemplo, a respeito da necessidade de suspender o acolhimento quando se torna impossível atender a todos. ${ }^{4}$ Ao promover o cuidado de usuários que sabidamente não 
precisam de atenção intensiva e poderiam se beneficiar de tratamento ambulatorial, mas não conseguem vaga imediatamente para isso, o CAPS assume que essa parcela de usuários ficaria desassistida e, ao mesmo tempo, centraliza a demanda ao se responsabilizar por pessoas cujo nível de atenção a se responsabilizar seria outro. ${ }^{4}$ Aqui é preciso apontar que a criação desta modalidade de atendimento ocorrem em função de uma falha da rede de atenção psicossocial pois, sem ter lugar o cuidado do usuário sem um quadro psiquiátrico grave ou situação intensa de sofrimento psíquico imediatamente para o cuidado, o CAPS assume a responsabilidade pela demanda sem que, até o momento da coleta, houvesse articulação com outros pontos da rede para oferecer diferentes modalidades de atenção em saúde mental.

Alguns autores trazem que $o$ conceito de acolhimento não se restringe apenas ao primeiro contato do usuário com o serviço, pois se desdobra em outras etapas: incluir a pessoa no serviço e realizar seguimento ou encaminhá-la para um serviço mais adequado para que ali cuidado transcorra. ${ }^{9}$ Deste modo, há uma responsabilização da equipe acolhedora pela trajetória do usuário na rede. O acolhimento serve como uma ponte entre os usuários e os serviços, e é a partir de como ocorre esse acolhimento que o usuário formará vínculo ou não com a instituição, por isso a importância de se pensar nessa estratégia. ${ }^{9-12}$ Essa visão corrobora com os resultados do atual estudo, partindo da mudança de como era feito o acolhimento, para o que passou a ser realizado.

Os entrevistados consideraram como fundamental o estabelecimento de um bom vínculo com os usuários e também as famílias. Segundo a visão dos entrevistados, é através do apoio às famílias que se consegue uma melhor adesão do usuário ao tratamento. Esse ponto-de-vista corrobora com a literatura ${ }^{12}$, que destaca que os CAPS têm os familiares como parceiros no tratamento; com isto, a família além de prestar auxílio aos usuários, tem o importante papel de incentivá-los no envolvimento com o projeto terapêutico. E foi percebendo a importância da família, que o CAPS teve como proposta a criação do grupo de acolhimento para os familiares. No entanto, é preciso destacar que a família não pode ser vista unicamente como um elo de ligação entre usuário e serviço, pois ela também deve ser alvo de cuidados por parte das equipes de saúde mental. ${ }^{1}$ 
Os resultados da pesquisa apontaram que os profissionais viam o trabalho em equipe como algo positivo dentro do serviço, por proporcionar uma maior troca de experiência, o compartilhamento de decisões e diferentes olhares sobre o mesmo objetivo. Esse compartilhar as decisões $^{6}$, as trocas entre os profissionais de diferentes áreas, incluindo desde os técnicos até a equipe de apoio do serviço, permitem um espaço de escuta e acolhimento a estes profissionais, e com isto, há uma melhora na acolhida da demanda da população.

É preciso apontar a falta de articulação entre os serviços da rede, pois a lógica do encaminhamento para o ambulatório de psiquiatria foi mencionada nas entrevistas, sem que a articulação com a atenção primária estivesse presente. Ao final da coleta de dados, no momento da devolutiva ao serviço, a equipe informou que o Grupo de Acolhimento fora desativado no CAPS para maior adesão à lógica do matriciamento em saúde mental na rede básica. Deste modo, podemos refletir que estão sendo articuladas discussões com equipes de outros serviços de saúde para o apoio matricial.

O acolhimento é fundamental para o cuidado integral em saúde ${ }^{13}$ e para as práticas afinadas com a política de humanização, as quais devem se fundamental na escuta qualificada e na responsabilização de usuário e profissionais pela saúde da população. Tal responsabilização só ocorre mediante o estreitamento do vínculo profissionalusuário, o trabalho em equipes de referência com características interdisciplinares e ações de apoio matricial.

A prática do acolhimento como diretriz operacional requer mudança no cuidado em saúde ${ }^{6}$, implicando valorização dos sujeitos envolvidos como protagonistas na produção de saúde; reorganização dos processos de trabalho, a partir da problematização das práticas cotidianas com discussões críticas e construtivas entre profissionais, usuários e gestores, disponíveis para mudança; aproximação da rede social; elaboração e acompanhamento dos Projetos Terapêuticos Singulares (PTS); postura de escuta que apresente compromisso com as necessidades do usuário, respeitando sua cultura, saberes e capacidade de avaliar riscos e construção coletiva com a Rede de Cuidados Integrais.

Tal qual os dados do presente artigo, a literatura da área ${ }^{4}$ também encontrou serviços nos quais diversos usuários que 
chegavam ao CAPS não eram demanda para este tipo de serviço, e o município não possuía outro serviço de atenção à saúde mental que os absorvesse. São exemplos, usuários que poderiam ter sido acolhidos na atenção primária e não em um serviço especializado, corria o risco de serem "desassistidas". 4 Ao assumir o cuidado das pessoas para as quais não se consegue encaminhamento, o CAPS centraliza a demanda, que deveria estar distribuída em serviços da atenção primária, como as entrevistas destacaram, por não terem perfil para atenção psicossocial.

Outro ponto relevante quanto ao acolhimento é seu papel no tocante às crises $^{14-15}$, pois com a expansão de serviços de base comunitária, é cada vez mais urgente $\mathrm{o}$ fortalecimento de práticas fundadas na construção de vínculo e cuidado integral, percebendo o sujeito em sofrimento e a criação de estratégias de atenção que se desvinculem do modelo médico-hospitalocêntrico baseado na remissão de sintomas.

\section{CONSIDERAÇÕES FINAIS}

O estudo teve como objetivo compreender o que os profissionais do CAPS entendiam por acolhimento e como este era realizado no serviço. Com a concretização desse artigo, considera-se que o objetivo foi alcançado. Inicialmente porque foi possível identificar as concepções dos profissionais de um CAPS sobre a ferramenta acolhimento, e em segundo lugar, pois foi possível perceber o processo de transformação do acolhimento ao longo do tempo, desencadeado por reflexões da equipe, passando de um processo feito em dupla, para uma prática grupal coordenada por um dos membros da equipe, em sistema de revezamento com outros.

Foi possível apreender o sentido e importância que os profissionais entrevistados agregam ao acolhimento, as dificuldades encontradas e a preocupação com a existência de vínculo equipeusuário-família no cuidado às pessoas em sofrimento psíquico. Ademais, o estudo também apontou para a necessidade de articulação entre serviços, no momento em que o acolhimento foi mantido enquanto prática grupal para assegurar que o usuário estivesse em atendimento enquanto uma vaga em outro serviço de saúde mental da cidade não fosse obtida. No entanto é preciso ressaltar que existe a necessidade de criação efetiva de um cuidado em rede, não apenas pela ampliação dos serviços existentes, mas especialmente para a implantação das ações de matriciamento 
em saúde mental junto à atenção básica, de forma que as pessoas sejam cuidadas e que o CAPS possa cumprir seu papel de articulador do território e de atenção às pessoas em situação mais intensa de sofrimento.

Destaca-se a necessidade de novos estudos na área, em diferentes localidades do país para problematizar o tema, bem como a investigação do acolhimento a partir da perspectiva de usuários e familiares.

\section{REFERÊNCIAS}

1. Ministério da Saúde (Brasil), Secretaria de Atenção à Saúde. Saúde Mental no SUS: os centros de atenção psicossocial. Brasília: Ministério da Saúde; 2004. [citado em 21 jan 2015]. 86p. Disponível em:

http://www.ccs.saude.gov.br/saude_mental /pdf/sm_sus.pdf.

2. Franco TB, Bueno WS, Merhy EE. O acolhimento e os processos de trabalho em saúde: o caso de Betim, Minas Gerais, Brasil. Cad. de Saúde Pública. 1999; 15(2):345-353.

3. Silva LG, Alves MS. O Acolhimento como Ferramenta de Práticas Inclusivas de Saúde. Rev. APS. 2008; 11(1):74-84.

4. Scheibel A, Ferreira LH. Acolhimento no CAPS: Reflexões Acerca da Assistência em Saúde Mental. Rev. Bahiana de Saúde Pública. 2011; 35(4):966-983.

5. Coimbra VCC, Kantorski LP. O Acolhimento num Centro de Atenção Psicossocial. Rev. Enferm. UERJ. 2005; 13:57-62.
6. Ministério da Saúde (Brasil), Secretaria de Atenção à Saúde, Núcleo Técnico da Política Nacional de Humanização. Acolhimento nas Práticas de Produção de Saúde. Brasília (DF): Ministério da Saúde; 2006. [citado em 19 jan 2015]. 44p. Disponível em: http://bvsms.saude.gov.br/bvs/publicacoes/ acolhimento_praticas_producao_saude.pdf.

7. Bardin L. Análise de conteúdo.

Lisboa: Edições 70; 2006. 281p.

8. Mendes EV. Editorial. REAS [internet]. 2013 [acesso em: 20 jan 2015]; 2 ESp2:1-3. Disponível em: http://www.uftm.edu.br/revistaeletronica/i ndex.php/enfer/article/download/552/416. 9. Schmidt MB, Figueiredo AN. Acesso, Acolhimento e Acompanhamento: Três Desafios para o Cotidiano as Clínica em Saúde Mental. Rev. Latinoam. Psicopat. Fund. 2009; 12(1): 130-140. 10. Gomes MCPA, Pinheiro R. Acolhimento e vínculo: práticas de integralidade na gestão do cuidado em saúde em grandes centros urbanos. Interface - Comunic, Saúde, Educ. 2005; 9 (17):287-301.

11. Jorge MSB, Pinto DM, Quinderé PHD, Pinto AGA, Sousa FSP, Cavalcante CM. Promoção da Saúde Mental Tecnologias do Cuidado: vínculo, acolhimento, co-responsabilização e autonomia. Ciência \& Saúde Coletiva. 2011, 16(7):3051-3060.

12. Silva L, Moreno V. A religião e a experiência do sofrimento psíquico: escutando a família. Ciência, Cuidado e Saúde. 2004; 3(2):161-168.

13. Ballarin MLGS, Carvalho FB, Ferigato SH, Miranda IMS, Magaldi CC. Centro de atenção psicossocial: convergência entre saúde mental e coletiva. Psic. em Estudo. 2011; 16(4):603611.

14. Zeferino MT, Cartana MHF, Fialho MB, Huber MZ, Bertoncello KCG.

Percepção dos trabalhadores de saúde sobre o cuidado às crises na Rede de 
Atenção Psicossocial. Esc. Anna Nery. 2016; 20(3):e20160059.

15. Silva MD. O cuidado na saúde pública: potencialidades de uma clínica em

RECEBIDO: 26/01/2016

APROVADO: 20/09/2016

PUBLICADO: $31 / 07 / 2017$ movimento. Est. Contemp. Subjetividade. 2016; 6(1):64-76. 